\title{
BENEFICIOS SOCIO AMBIENTALES POR POTABILIZACIÓN DEL AGUA EN LOS PUEBLOS PALAFÍTICOS DE LA CIÉNAGA GRANDE DE SANTA MARTA-COLOMBIA
}

\section{ENVIRONMENTAL SOCIAL BENEFITS FOR WATER POTABILIZATION IN THE PALAFITIC PEOPLES OF CIENAGA GRANDE DE SANTA MARTA-COLOMBIA}

\author{
José Luis Lugo ${ }^{1}$, Elkyn Rafael Lugo²
}

\begin{abstract}
${ }^{1}$ Ingeniero Ambiental y Sanitario, Magister en Ingeniería Ambiental. Universidad del Norte, km 5 vía Puerto Colombia, Barranquilla - Atlántico, Colombia, Calle 30 con Cra 19a, Mz. 91 casa 8, Urb. El Pando, Santa Marta - Magdalena, Colombia, e-mail: jllugo@uninorte.edu.co; ${ }^{2}$ Economista, Magister en Economía. Universidad del Norte, Barranquilla, Colombia. Km 5 vía Puerto Colombia, Barranquilla - Atlántico, Colombia. Calle 30 con Cra 19a, Mz. 91 casa 8, Urb. El Pando, Santa Marta Magdalena, Colombia, e-mail: elkinlugo@gmail.com
\end{abstract}

Rev. U.D.C.A Act. \& Div. Cient. 21(1): 259-264, Enero Junio, 2018

https://doi.org/10.31910/rudca.v21.n1.2018.685

\section{INTRODUCCIÓN}

La purificación del agua comprende el uso de tecnologías, en las que se llevan a cabo diversos procesos de tratamiento, cuya finalidad es la de remover contaminantes en el agua hasta ciertas concentraciones, que no representen riesgo para la salud humana (Rojas, 2015). Un sistema de tratamiento no convencional de agua potable hace referencia a tratamientos alternativos, bien sea por procesos avanzados de potabilización o por sistemas descentralizados o emergentes.

La implementación de sistemas de tratamiento de agua potable requiere de inversiones económicas; sin embargo, existen muchas comunidades rurales, en el mundo, que no cuentan con los recursos económicos para la construcción de tecnologías de purificación de agua, lo cual, constituye un riesgo considerable para la salud de las personas, que viven en estas zonas (Arango, 2004). Gruber et al. (2013) y Fabiszewski De Aceituno et al. (2012), en sus estudios, por ejemplo, evidencian el gran reto que tienen las comunidades rurales en lograr el tratamiento de agua, para el consumo humano seguro.

Debido a esta problemática, se han empleado tecnologías eficientes a bajo costo, para la potabilización artesanal del agua en comunidades rurales; sin embargo, las comunidades palafíticas de la Ciénaga Grande de Santa Marta (CGSM), Colombia, específicamente, Nueva Venecia y Buena Vista, que son veredas palafíticas, en donde las casas están construidas de maderas y localizadas sobre el complejo cenagoso, carecen de sistemas de distribución de agua potable y alcantarillado (CEP, 2011; Narváez et al. 2008). A pesar de esta falta de servicios básicos, no se cuenta con estudios que indiquen el grado de contaminación del agua que consumen estas poblaciones, ni su relación con las probables enfermedades contraídas, por la falta de agua potable.

El objetivo principal de esta indagación básica fue analizar, desde el punto de vista socio-ambiental, los beneficios asociados a la implementación de dos alternativas de potabilización del agua, en Nueva Venecia y en Buena Vista.

\section{MATERIALES Y MÉTODOS}

El área de estudio comprende dos corregimientos de Sitio Nuevo (Magdalena): Nueva Venecia (latitud de $10^{\circ} 49^{\prime} \mathrm{N}$; longitud de $74^{\circ} 34^{\prime} \mathrm{O}$ ) y Buena Vista (latitud de $10^{\circ} 50^{\prime} \mathrm{N}$; longitud de $74^{\circ} 30^{\prime}$ O), están ubicados en la Ciénaga de Pajarales o Complejo Pajarales (CP), contigua a la Ciénaga Grande de Santa Marta (CGSM). El tipo de estudio de este reporte de caso es indagatorio, en el que, a través de una encuesta y un muestreo de calidad de agua, se diagnostica la problemática relacionada con la gestión del agua potable, en los dos pueblos palafíticos mencionados y, además, se realiza un análisis de beneficio socio-ambiental de dos alternativas de potabilización a bajo costo, que se desarrollarán en una futura investigación.

El análisis socio-ambiental, se realizó por medio de encuestas aplicadas en los pueblos de estudio; se indagó sobre la gestión del agua de consumo humano en las viviendas. La información obtenida, mediante el instrumento aplicado, se relacionó con la revisión de artículos científicos que han pu- 
blicado resultados del tratamiento descentralizado, de agua potable propuesto.

Las encuestas fueron aplicadas en las viviendas, partiendo de una población de 300 casas, en Nueva Venecia y 150, en Buena Vista. Se analizaron, como muestra, a personas mayores de 18 años de diferentes hogares, con un total de 20, en Nueva Venecia y 18, en Buena Vista, el 21 de febrero de 2017, mediante un muestreo aleatorio simple. En este instrumento, se aplicaron preguntas sencillas, dicotómicas y abiertas, adaptadas al bajo nivel de formación de las personas (Tabla 1), las cuales, fueron validadas por cuatro investigadores expertos en potabilización de agua.
Con base a la información recolectada en campo, a través de las encuestas, se identificaron las necesidades y las preferencias de las comunidades palafíticas estudiadas, relacionadas con la gestión del agua de consumo humano. Estos aspectos fueron comparados con lo que se esperaría del tratamiento de agua propuesto. Finalmente, se realizó el análisis de beneficio socio-ambiental, asociado al proyecto propuesto de gestión social de purificación de agua.

Adicionalmente, se llevaron a cabo dos muestreos puntuales de calidad de agua en el punto de captación de agua (caño Aguas Negras), de los dos pueblos palafíticos estudiados, ubicado a $10^{\circ} 48^{\prime} 41.01^{\prime \prime}$ de latitud Norte y $74^{\circ} 36^{\prime} 21.21^{\prime \prime}$ de

Tabla 1. Percepción y gestión del agua de consumo de los habitantes de los pueblos palafíticos, en porcentaje (\%).

\begin{tabular}{|c|c|c|c|c|}
\hline \multirow{3}{*}{ Pregunta } & \multicolumn{4}{|c|}{ Pueblo } \\
\hline & \multicolumn{2}{|c|}{ Nueva Venecia } & \multicolumn{2}{|c|}{ Buena Vista } \\
\hline & Si & No & Si & No \\
\hline $\begin{array}{l}\text { 1. ¿Considera que el agua que consume le ha ocasionado problema de } \\
\text { salud (enfermedades)? }\end{array}$ & 95,0 & 5,0 & 77,8 & 22,2 \\
\hline ¿Qué síntomas se le ha presentado con el agua que consume? & \multicolumn{2}{|c|}{$\begin{array}{l}\text { Diarrea }\left(61,1^{\mathrm{b}}\right) \text {, vómito } \\
\left(33,3^{\mathrm{b}}\right) \text {, dolor de estómago } \\
\left(44,4^{\mathrm{b}}\right) \text { y fiebre }\left(5,5^{\mathrm{b}}\right)\end{array}$} & \multicolumn{2}{|c|}{$\begin{array}{l}\text { Diarrea }\left(70,0^{\mathrm{b}}\right) \text {, vómito } \\
\left(55,0^{\mathrm{b}}\right) \text {, dolor de estómago } \\
\left(45,0^{\mathrm{b}}\right) \text { y fiebre }\left(10,0^{\mathrm{b}}\right)\end{array}$} \\
\hline 2. ¿Cree que el agua que consume es de buena calidad? & 0,0 & 100,0 & 5,6 & 94,4 \\
\hline $\begin{array}{l}\text { 3. ¿Cree que el agua que consume requiere de tratamiento para ser } \\
\text { bebida? }\end{array}$ & 100,0 & 0,0 & 94,4 & 5,6 \\
\hline $\begin{array}{l}\text { 4. ¿Estaría dispuesto a recibir capacitación de cómo realizar } \\
\text { tratamiento del agua que consume en casa? }\end{array}$ & 100,0 & 0,00 & 100,0 & 0,0 \\
\hline $\begin{array}{l}\text { 5. ¿Estaría dispuesto a tratar su agua que consume o su creencia no le } \\
\text { permite hacerlo? }\end{array}$ & 100,0 & 0,0 & 100,0 & 0,0 \\
\hline 6. ¿Utiliza algún tipo de tratamiento para el agua que consume? & 80,0 & 20,0 & 44,4 & 55,6 \\
\hline $\begin{array}{l}\text { 7. ¿Conoce técnicas a bajo costo de tratamiento de agua potable en } \\
\text { hogares? }\end{array}$ & 90,0 & 10,0 & 83,3 & 16,7 \\
\hline ¿Qué técnicas de tratamiento de agua potable conoce? & \multicolumn{4}{|c|}{$\begin{array}{c}\text { Cloración, cloración más SODIS, calentamiento de agua, } \\
\text { filtros comerciales y de tela }\end{array}$} \\
\hline $\begin{array}{l}\text { 8. ¿Cree necesario realizar inversiones para purificar el agua que } \\
\text { consume? }\end{array}$ & 100,0 & 0,0 & 100,0 & 0,0 \\
\hline $\begin{array}{l}\text { 9. ¿Ha recibido capacitación sobre temas de prevención de } \\
\text { enfermedades relacionadas con el agua? }\end{array}$ & 50,0 & 50,0 & 50,0 & 50,0 \\
\hline $\begin{array}{l}\text { 10. ¿Ha recibido donaciones para tratar el agua por parte del gobierno } \\
\text { u otras organizaciones? }\end{array}$ & 35,0 & 65,0 & 27,8 & 72,2 \\
\hline 11. ¿Le hace tratamiento al agua que usa para baño? & 40,0 & 60,0 & 11,1 & 88,9 \\
\hline 12. ¿Le hace tratamiento al agua que usa para cocina? & 70,0 & 30,0 & 22,2 & 77,8 \\
\hline 13. ¿Le hace tratamiento al agua residual (desperdicio)? & 0,0 & 100,0 & 0,0 & 100,0 \\
\hline ¿Qué se hace con el agua residual? & \multicolumn{2}{|c|}{ Se vierte a la ciénaga ${ }^{a}$} & \multicolumn{2}{|c|}{ Se vierte a la ciénaga ${ }^{a}$} \\
\hline
\end{tabular}

${ }^{a}$ Frecuencia porcentual del $100 \%$.

${ }^{\text {b }}$ Porcentaje total de percepción (no suma el 100\% porque varios encuestados percibieron múltiples síntomas). 
longitud Oeste. El primer muestreo, se llevó a cabo a finales de marzo y el segundo, a finales de abril de 2017. Se analizaron dos parámetros de calidad de agua, en el laboratorio de Calidad de Aguas, de la Universidad del Norte, siguiendo los lineamientos de Standard Methods for the Examination of Water and Wastewater: Turbiedad (2130, Nefelométrico) y Coliformes totales (9222 B, Filtración por membrana).

\section{RESULTADOS Y DISCUSIÓN}

Encuesta de percepción de la calidad de agua: En los dos pueblos estudiados, se encontró que todos los encuestados consideraron lo siguiente: que el consumo directo del agua de río podría ocasionar enfermedades; estarían dispuestos a recibir capacitación para potabilizar agua en la vivienda, así como a tratar el agua sin ningún tipo de restricción relacionada con creencias o aspectos culturales; creyeron necesario realizar inversiones para la potabilización del agua y que el agua de desperdicio de cocina y de baño se vierte directamente al complejo cenagoso.

Otro aspecto a resaltar es que la mayoría de las personas en estos dos pueblos consideraron que el agua que consumen es de mala calidad, ya que han padecido enfermedades que las relacionan con su consumo. De manera general, los síntomas que más percibieron fueron los siguientes: diarrea, vómito, dolor de estómago y fiebre, siendo la diarrea la más frecuente.

Lo anterior es consistente con lo reportado en el puesto de salud de Bocas de Aracataca, localizado en las inmediaciones de los pueblos palafíticos, ya que, en 2004 , las infecciones respiratorias agudas (IRA) y las enfermedades diarreicas agudas (EDA) fueron las más frecuentes, reportadas con un 60 y $30 \%$, respectivamente, estando relacionadas, principalmente, con la falta de agua potable y de servicios de alcantarillado y aseo (CEP, 2011).

Aunque muchos de los encuestados contestaron que sí conocen técnicas de tratamiento de agua que se podrían implementar a bajo costo en los hogares, como la cloración, la cloración más la desinfección solar (SODIS), el calentamiento de agua, los filtros comerciales de membrana y de tela, no se tiene un enfoque integrado para la potabilización del agua, al no conocer ni emplear una serie de procesos consecutivos para este fin.

Un agua turbia con presencia de microorganismos patógenos, como la de la fuente de captación de agua de estos pueblos, en la que se encontró que la turbiedad varió entre 630 y 650 Unidades Nefelométricas de Turbiedad (NTU) y los coliformes totales entre 6.500 y 8.500 Unidades Formadoras de Colonia en $100 \mathrm{~mL}$ (UFC/100mL), requiere una serie de procesos para la remoción de contaminantes del agua, como por ejemplo, el método de barreras múltiples, propuesto por CAWST (2009), para el tratamiento de agua en hogares.

Análisis de beneficio socio-ambiental: Entre las técnicas que más utilizan en estas dos comunidades rurales está la cloración, la cual, requiere de un personal capacitado para su aplicación en el tratamiento de agua potable, ya que su dosificación requiere de mucho cuidado, puesto que el cloro residual en el agua tratada puede formar compuestos organoclorados, conocidos como trihalometanos, que se han identificado como cancerígenos para la salud humana (Arjona et al. 2012).

Las otras técnicas empleadas por estas comunidades: calentamiento de agua, filtros comerciales y de tela, también poseen desventajas al emplearse de manera aislada, es decir, sin combinaciones con otras para la potabilización de agua. La cloración y SODIS no funcionan en un agua turbia, por lo que se requiere complementar estos sistemas con pretratamientos, basados en la sedimentación o filtración de partículas (Rojas, 2015).

Por las desventajas o problemas mencionados, se propone un sistema de tratamiento del agua, que abarca los siguientes procesos y operaciones: (1) coagulación (coagulantes naturales: Moringa oleífera (Moringa) y Cassia fistula (Cañandonga); (2) filtración (filtro de bioarena y de carbón activado) y (3) desinfección (SODIS y lámpara UV) (Figura 1).

En el proceso de coagulación-floculación, mediante coagulantes naturales, se obtienen beneficios importantes en comparación con el uso de coagulantes químicos. Los coagulantes químicos, como las sales de aluminio, pueden causar problemas de Alzheimer, síndromes de demencia y disminución de la capacidad motora y mental (Okuda et al. 2001); resultan más costosos que los naturales (Betatache et al. 2014), imposibilitándose su aplicación en comunidades con bajos recursos económicos y, además, generan mayor cantidad de lodos (Jodi et al. 2012), que afectan a la salud humana y al medio ambiente.

Los coagulantes naturales han sido igual de eficientes en la clarificación del agua como los coagulantes químicos; pero a diferencia de estos últimos, los naturales se consideran seguros para la salud humana y ambientalmente amigables, debido a su característica de biodegradabilidad (Ramírez \& Jaramillo, 2015), propiedad que lo hace potencial para preservar y proteger el medio ambiente, como, por ejemplo, los cuerpos de agua y la biota acuática.

Por otra parte, los filtros de bioarena son de gran aplicabilidad en la potabilización de agua, ya que son eficaces para remover turbiedades del agua (Raza el al. 2015) y microrga- 

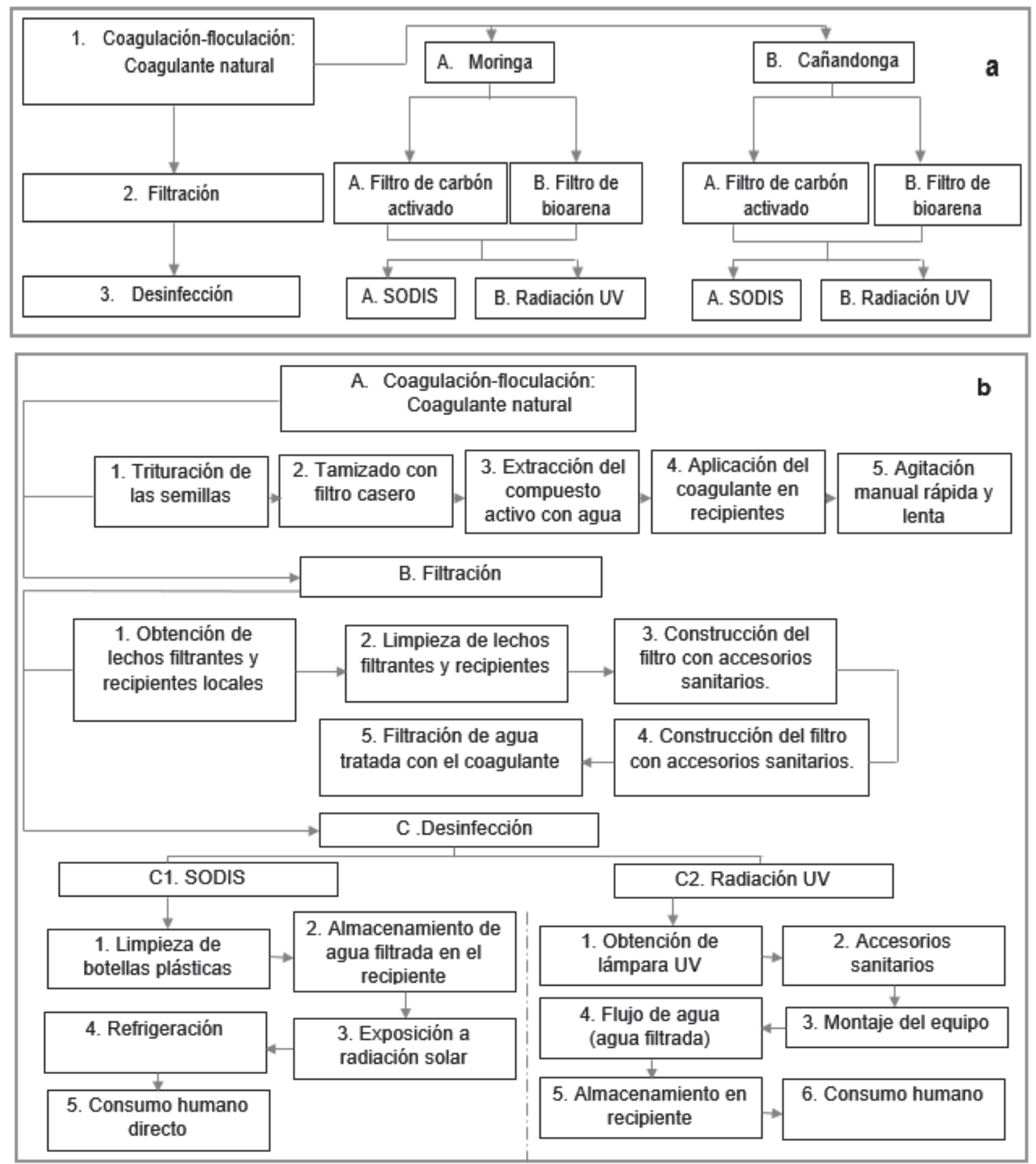

Figura 1. (a) Diagrama general de los procesos propuestos para la potabilización no convencional (artesanal) y (b) Esquema general del procedimiento de purificación de agua en los pueblos palafícos: Nueva Venecia y Buena Vista.

nismos, como bacterias (Segalla et al. 2015) y virus (YoungRojanschi \& Madramootoo, 2014). Además, se ha demostrado que hogares han reducido las tasas de incidencia de enfermedades diarreicas, como lo reporta Stauber et al. (2012), enfermedad que ha cobrado millones de vidas en el mundo (OMS, 2015). A pesar de estos estudios, en los dos pueblos palafíticos analizados, se evidenció que, en todos los hogares encuestados, no empleaban este tipo de filtro comúnmente usado en el mundo, que podría ocasionar un impacto positivo en la salud de las personas. 
Otro tipo de filtro considerado es el de carbón activado, porque esta capa filtrante en el tratamiento de agua potable posee los siguientes atributos: elimina olor, color, sustancias químicas y bacterias. Entre las sustancias químicas, se reducen los compuestos hidrocarbonados clorados, nitrobencenos, aldehídos y alcanos (Lluíz, 2008). Dadas estas propiedades, con este lecho, se podría beneficiar a las poblaciones de estudio, ya que las fuentes de aguas superficiales de acceso a Nueva Venecia y a Buena Vista están recibiendo aporte de contaminantes de diferentes industrias, lo cual, requerirían de tecnologías, como la del carbón activo, que permitan su respectiva remoción, pero para ello, se necesitarían estudios pertinentes.

Al aplicar los procesos antes descritos, se esperaría un agua muy clarificada, pero en los procesos de filtración, por ejemplo, en el filtro de bioarena y de carbón activado, no se remueve la totalidad de los microorganismos patógenos (Young-Rojanschi \& Madramootoo, 2014), por tanto, un proceso de desinfección sería acertado, en este caso, por lo que se proponen dos técnicas de desinfección: (1) SODIS, que se utiliza en algunos de los hogares y (2) Luz UV mediante lámparas UV-C. La primera requiere de alta intensidad de luz solar y, la segunda, de una lámpara UV, con una longitud de onda de $254 \mathrm{~nm}$, la cual, es considerada como la más efectiva para desactivar el ADN de los microorganismos (González-Púmariega et al. 2009).

Estas dos tecnologías han demostrado reducir el riesgo de la propagación de enfermedades relacionadas con el agua contaminada (Gruber et al. 2013); sin embargo, se deben manejar con mucho cuidado, por ejemplo: SODIS y UV no funcionan con aguas turbias (Abd-Elmaksoud et al. 2013); SODIS es ineficiente para eliminar aquellos microorganismos que forman esporas (Boyle et al. 2008) y baja su rendimiento en cantidades de volúmenes de agua (Garrido et al. 2013).

Es por ello que, para lograrse un tratamiento adecuado de potabilización en las dos comunidades estudiadas, teniendo en cuenta los sistemas propuestos en este trabajo, se requiere capacitación en los hogares, aunque las técnicas sean simples, así como la gestión de la administración gubernamental, para poder llevar a cabo estos proyectos sociales, que benefician a la población. Finalmente, las técnicas expuestas en esta investigación podrían ser eficientes para solucionar esta problemática socio-ambiental, pero se necesita de estudios que den información de la viabilidad técnica para la potabilización de agua, mediante los sistemas a bajo costo, propuestos.

Agradecimientos: Los autores agradecen a COLCIENCIAS, al grupo de Investigación Suelo, Ambiente y Sociedad de la Universidad del Magdalena y a la Gobernación del Magdalena. Conflicto de intereses: Este manuscrito fue elaborado y revisado con la participación de todos los autores, quienes declaramos no tener conflicto de intereses, que ponga en riesgo la validez de los resultados. Financiación: El presente estudio surgió de un proyecto que se realizó con apoyo financiero de COLCIENCIAS y de la Gobernación del Magdalena.

\section{BIBLIOGRAFÍA}

1. ABD-ELMAKSOUD, S.; NARANJO, J.E.; GERBA, C.P. 2013. Assessment of a portable handheld UV light device for the disinfection of viruses and bacteria in water. Food and Environmental Virology (Estados Unidos). 5(2):8790.

2. ARANGO, Á. 2004. La biofiltración, una alternativa para la potabilización del agua. Rev. Lasallista Invest. (Colombia). 1(2):61-66.

3. ARJONA, S.; TORRES, P.; CRUZ, C.; LOAIZA, D.; ESCOBAR, J. 2012. Efecto del punto de precloración sobre la formación de Trihalometanos en procesos convencionales de potabilización de agua. Rev. Ingenierías Universidad de Medellín (Colombia). 11(20):57-66.

4. BETATACHE, H.; AOUABED, A.; DROUICHE, N.; LOUNICI, H. 2014. Conditioning of sewage sludge by prickly pear cactus (Opuntia ficus indica) juice. Ecol. Eng. (Holanda). 70:465-469.

5. BOYLE, M.; SICHEL, C.; FERNÁNDEZ-IBAÑEZ, P.; ARIAS-QUIROZ, G.B.; IRIARTE-PUÑA, M.; MERCADO, A.; UBOMBA-JASWA, E.; MCGUIGAN, K.G. 2008. Bactericidal effect of solar water disinfection under real sunlight conditions. Appl. Environ. Microbiol (Estados Unidos). 74(10):2997-3001.

6. Centre for Affordable Water and Sanitation Technology -CAWST-. 2009. Biosand Filter. Manual for Design, Construction, Installation and Maintenance. Disponible desde Internet en: https://www.calvin.edu/academic/engineering/seniordesign/SeniorDesign09-10/ team02/web/Biosand_Manual_Spanish.pdf (con acceso 05/05/17).

7. CEP - Banco de la República. 2011. La economía de las ciénagas del Caribe colombiano. Ed. Banco de la Republica. 197p.

8. FABISZEWSKI DE ACEITUNO, A.M.; STAUBER, C.E.; WALTERS, A.R.; MEZA SANCHEZ, R.E.; SOBSEY, M.D. 2012. A randomized controlled trial of the plastic-housing BioSand filter and its impact on diarrheal disease in Copan, Honduras. Am. J. Trop. Medicine and Hygiene. 86(6):913-921. 
9. GARRIDO, I.; FERNÁNDEZ, R.; STORACI, V. 2013. Evaluación del método "SODIS" en la desinfección del agua para abastecimiento en La Guadalupe, Chirgua, municipio Bejuma del estado Carabobo. Rev. Ingeniería UC (Venezuela). 20(2):29-38.

10. GONZÁLEZ-PÚMARIEGA, M.; VIERNHES, M.; SÁNCHEZ-LAMAR, Á. 2009. La radiación ultravioleta. Su efecto dañino y consecuencias para la salud humana. Theoria (Chile). 18(2):69-80.

11. GRUBER, J.; REYGADAS, F.; ARNOLD, B.; RAY, I.; NELSON, K.; COLFORD, J. 2013. Stepped wedge, cluster-randomized trial of a household UV-disinfection and safe storage drinking water intervention in rural Baja California sur, Mexico. Am. J. Trop. Med. Hygiene. 89(2):238-245.

12. JODI, M.L.; BIRNIN-YAURI, U.A.; YAHAYA, Y.; SOKOTO, M.A. 2012. The use of some plants in water purification. Global Adv. Res. J. Chem. Mater. Sci. (Estados Unidos). 1(4):71-75.

13. LLUÍZ, J. 2008. La problemática del tratamiento del agua potable. Medicina Naturista (España). 2(2):6975.

14. NARVÁEZ, S.; GÓMEZ, M.; ACOSTA, J. 2008. Coliformes termotolerantes en aguas de las poblaciones costeras y palafíticas de la Ciénaga Grande de Santa Marta, Colombia. Acta Biol. Col. 13(3):113-122.

15. OKUDA, T.; BAES, A.U.; NISHIJIMA, W.; OKADA, M. 2001. Isolation y caracterization of coagulant extracted from Moringa oleifera seed by salt solution. Water Research. 35: 405-410.
16. Organización Mundial de la Salud -OMS-. 2015. Disponible desde Internet en: http://www.who.int/mediacentre/factsheets/fs391/es/ (con acceso 20/08/16).

17. RAMÍREZ, H.; JARAMILLO, J. 2015. Agentes naturales como alternativa para el tratamiento del agua. Rev. Fac. Cienc. Básicas (Colombia). 11(2):136-153.

18. RAZA, S.; REHMAN, A.; HUSSAIN, F.; NASIR, A.; CHAND, K. 2015. Design of a slow sand bed filtration system for purification of canal water. QUEST (Estados Unidos). 14(1):14-18.

19. ROJAS, J.A. 2015. Purificación del agua. Ed. Escuela Colombiana de Ingeniería (Colombia). 473p.

20. SEGALLA, B.; SOARES, M.; ROMERO, L.; SENS, M. 2015. Comparison of backwashing with conventional cleaning methods in slow sand filters for small-scale communities. Desalin. Water Treatm. (Italia). 54(1):1-7.

21. STAUBER, C.E.; PRINTY, E.R.; MCCARTY, F.A.; LIANG, K.R.; SOBSEY, M. D. 2012. Cluster randomized controlled trial of the plastic biosand water filter in Cambodia. Environ. Sci. Technol. 46(2):722-728.

22. YOUNG-ROJANSCHI, C.; MADRAMOOTOO, C. 2014. Intermittent versus continuous operation of biosand filters. Water Research. 49:1-10.

Recibido: Septiembre 8 de 2018

Aceptado: Febrero 20 de 2018

Cómo citar:

Lugo, J.L.; Lugo, E.R. 2018. Beneficios socio ambientales por potabilización del agua en los pueblos palafíticos de la ciénaga grande de Santa Marta-Colombia. Rev. U.D.C.A Act.\& Div. Cient. 21(1): 259-264. 